PREPRINT

AS54

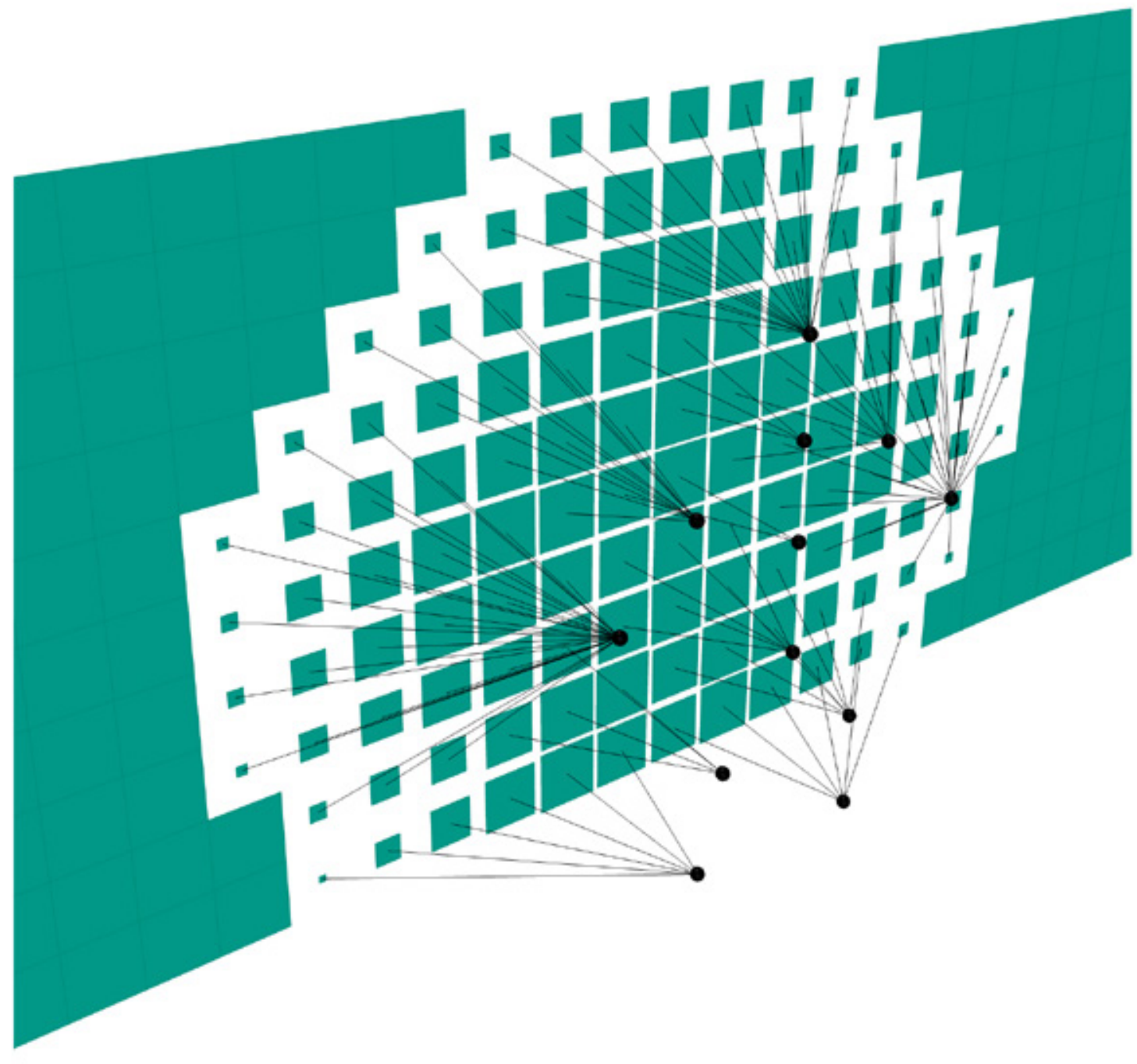




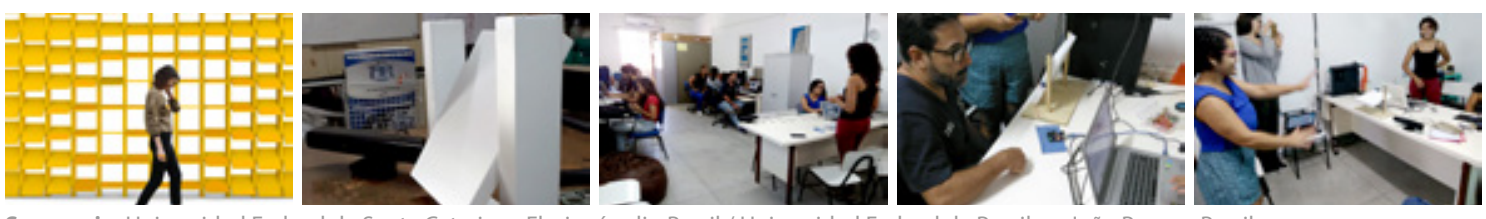

Secuencia: Universidad Federal de Santa Catarina - Florianópolis, Brasil / Universidad Federal de Paraiba - João Pessoa, Brasil Fotos: Jéssica Carvalho / Carlos Nome / Natália Queiroz

\title{
PARED CINÉTICA: UNA FACHADA HUMANA Y AMBIENTALMENTE RESPONSIVA. UN ENFOQUE DE EDUCACIÓN EN ARQUITECTURA'
}

\author{
Natália de Queiroz Nome², Jessica M. Carvalho³, Carlos Eduardo Verzola Vaz ${ }^{4}$, Carlos \\ Alejandro Nome ${ }^{5}$
}

RESUMEN

\begin{abstract}
Este artículo presenta la estructura conceptual (framework) del proyecto y del prototipo funcional de una pared cinética que responde a las necesidades de los seres humanos en términos de confort térmico y de la relación con la trayectoria solar. La descripción de la estructura conceptual es construida como un ejercicio potencialmente replicable en escuelas de arquitectura en América Latina. El estudio demuestra aproximaciones entre temas como "arquitectura responsiva", modelaje paramétrico y algorítmico en el proyecto de envolturas arquitectónicas. El resultado es un prototipo funcional de bajo costo, como también la descripción de la estructura conceptual de un ejercicio de diseño edilicio, posteriormente discutido en un grupo focal con estudiantes de graduación y posgrado. El abordaje contribuye pedagógicamente a la discusión de la complejidad de los proyectos de edificios y a la introducción de la automatización, el modelaje paramétrico y la programación en ejercicios de diseño contemporáneo.
\end{abstract}

Palabras clave: arquitectura responsiva, educación, fachada cinética, modelaje paramétrico, sombreamiento.

\section{ABSTRACT}

The paper presents the framework of the design and functional prototype of a perforated panel wall responsive to human movements and solar trajectory, as a replicable exercise for architecture schools in Latin America. The study demonstrates approximations between themes such as responsive architecture, parametric modeling associated to algorithmic modeling in the design of envelope elements. The result is a low cost functional prototype and the Framework for a replicable exercise, that was discussed in a focal group with undergraduate and graduate students. It contributes pedagogically to discuss the complexity of building envelope design and the introduction of automation, parametric modeling and programming in contemporary design exercises.

Keywords: responsive architecture, education, kinetic facade, parametric modeling, shading.

Artículo recibido el 10 de septiembre de 2018 y aceptado el 19 de diciembre de 2018 DOI: https://doi.org/10.22320/07196466.2018.36.054

[1] El presente trabajo fue realizado con el apoyo de la Fundación de Amparo a la Investigación e Innovación del Estado de Santa Catarina.

[2] Departamento de Arquitectura y Urbanismo, Universidad Federal de Santa Catarina, Florianópolis, Brasil. nataliaqueiroz@labcon. ufsc.br

[3] Departamento de Arquitectura y Urbanismo, Universidad Federal de Santa Catarina, Florianópolis, Brasil. jessica.mcarvalho@

hotmail.com

[4] Departamento de Arquitectura y Urbanismo, Universidad Federal de Santa Catarina, Florianópolis, Brasil.cevv00@gmail.com

[5] Departamento de Arquitectura y Urbanismo, Universidad Federal de Paraíba, João Pessoa, Brasil.carlos.nome@gmail.com 
El desarrollo de sistemas de envolturas es inherentemente complejo, pues responde a múltiples fenómenos naturales y genera transición entre el exterior y el interior de un edificio. Además de la protección contra agentes del medio ambiente (contaminación, lluvia, viento, etc.), consideraciones como la estética del sistema de envoltura, el confort (ofuscación, temperatura, calidad del aire, etc.) y, consecuentemente, el desempeño energético y el impacto ambiental son fundamentales, en este marco (JAGGER, Robert W, 2016). La exploración de soluciones proyectuales para sistemas complejos, como el aquí postulado, puede beneficiarse de la integración con procesos computacionales. Al adicionar las oportunidades que provienen de una "arquitectura responsiva", asociada a una expectativa de performance declarada, esta integración se torna imprescindible.

Conceptos como el de arquitectura responsiva proponen gestionar cuestiones entre usuario, comodidad $y$ seguridad, a partir del uso de sensores, flujos de información y nubes de datos (Beesley y Khan, 2009). Desde esa aproximación, se puede generar una arquitectura mutable o con un diseño basado en el performance (Oxman, 2008, Beesley y Khan, 2009). El proyecto de envolturas es uno de los sistemas presente en proyectos de edificaciones contemporáneas en que son incorporados aspectos de aplicación del mencionado concepto. Kroner (1997) muestra, ciertamente, una serie de aspectos preponderantes para una arquitectura en estos términos. En lo que se refiere a envoltura, es posible señalar la contemplación de aspectos como sensibilidad a la presencia humana, control de luz, calor y ventilación.

En las últimas décadas, el proyecto de la envoltura de los edificios se viene dividiendo en dos enfoques: la pasiva, sin uso de energía eléctrica, y la activa, con uso de energía eléctrica, que utiliza un enfoque de innovación basado en la tecnología (Fox, 2016). Las herramientas computacionales como las de simulación y de programación visual, así como la popularización de la fabricación digital y de la automatización de edificaciones, han facilitado las etapas de prototipado de esos enfoques. A pesar del escenario favorable a la investigación de estas aplicaciones, generalmente estas soluciones son vistas como onerosas y de poca accesibilidad técnica. Por diversos motivos, estrategias activas que integran diseño basado en performance todavía son poco exploradas en el contexto de las escuelas de arquitectura, principalmente las latinoamericanas. Esta situación contrasta con la creciente popularización de laboratorios de fabricación digital (Sperling, 2015) que permitirían desarrollo de investigaciones de esa naturaleza. En este sentido, vale la pena preguntarse ¿cómo incluir los contenidos sobre modelado algorítmico, programación y automatización, en el contexto de enseñanza en escuelas de arquitectura y urbanismo en América Latina, la que aún se encuentra mayormente basada en métodos y herramientas tradicionales? 
Para ilustrar el escenario latinoamericano referido, se llevó a cabo una búsqueda en CumInCAD, utilizando los términos "educación" y "programación" en inglés, español y portugués. Después se repitió la búsqueda empleando los términos "educación" y "automatización". En la base de datos, que consideró los últimos cinco años, fueron encontrados, en la primera búsqueda, 636 artículos en inglés, 12 en portugués y 20 en español. Para la segunda búsqueda los resultados fueron 528 en inglés, 6 en portugués y 18 en español. Esa información no indica necesariamente falta de producción latinoamericana en el área, pero sí una producción menos expresiva para el contexto local. Desde esa perspectiva, se vuelve de gran importancia caracterizar estructuras conceptuales y experiencias aplicadas en el mundo latinoamericano para la difusión de diferentes enfoques en esta región.

El presente artículo tiene por objetivo exponer la estructura conceptual (framework) de un prototipo funcional de una envoltura formada por elementos huecos "responsivos" a los movimientos humanos y a la trayectoria solar, presentado como ejercicio replicable en escuelas de arquitectura en América Latina. De esta manera, se postula una oportunidad de aproximación entre la temática de proyectos de envolturas responsivas y la enseñanza de arquitectura en Latinoamérica. Para ejemplificar una aplicación, el artículo plantea los resultados de un estudio piloto elaborado con alumnos de graduación y postgrado de arquitectura. Dicho proyecto parte de la búsqueda de una fachada interactiva, que permitiría visibilidad al exterior, con la aproximación de un usuario, pero que también impidiera la entrada y visión directa del sol en el interior del espacio abrigado. Esta interactividad, evitaría fenómenos involuntarios perjudiciales a la actividad humana, como el deslumbramiento, por ejemplo. En concreto, la pared está compuesta por módulos pivotantes que son accionados por la hora solar y el movimiento humano. El accionamiento por la hora solar ocurre de forma independiente de la acción humana y responde a la definición de un algoritmo específico. Por medio de sensores ópticos, la detección de la aproximación del usuario acciona parte de los módulos que pasan a obedecer al contorno de los movimientos humanos.

\section{Una breve contextualización del uso de métodos digitales en las escuelas de arquitectura}

Según Oxman (2008), el conocimiento asociado al diseño digital constituye un conjunto nuevo de conceptos. Este conjunto incluye ideas relacionadas al significado de la forma, la naturaleza del conocimiento funcional y formal, los modelos de procesos generadores, así como la integración con datos, desempeño y técnicas interactivas. Así siendo, es importante presentar estrategias pedagógicas y describir aplicaciones que acomodan esta base de conocimiento.

Kotnik (2010), por su parte, indica que los métodos de proyecto auxiliados por computador y asociados a la programación permiten una mejor comprensión de los conceptos matemáticos y su interacción con elementos del proyecto. Como tal, el computador no es una herramienta neutra, sino un medio activo para ayudar a los métodos de diseño. Este medio puede ser un facilitador para la aplicación de conceptos científicos de forma práctica y teórica. Para el mismo autor, los problemas de adaptabilidad e inercia del sistema educativo se dividen en tres clases de influencia: el medio de diseño; el conocimiento y la teoría; y los modelos y metodologías. El cambio de los métodos digitales recientes impregna aquellas tres clases. En término de enseñanza, Kotnik traza cuatro pasos didácticos básicos para abordar los cambios digitales:

Conceptualizar y definir un tipo específico de "material digital" (objetivo)

Definir un modelo específico de diseño digital relacionado con la formación, generación, rendimiento, o con las relaciones entre distintos componentes de modelos digitales (prototipo digital).

Seleccionar un contexto que pueda demostrar mejor el comportamiento y la aplicabilidad del "material de diseño" en relación a los principios asociados con la formación, la generación y el rendimiento (localidad).

Desarrollar y presentar una taxonomía (vinculada con la arquitectura digital) que se pueda utilizar para describir los procesos de pensamiento del diseño arquitectónico digital.

\section{Arquitectura cinética aplicada al proyecto de envolturas}

En las últimas décadas, el concepto de arquitectura cinética viene tomando mayor espacio en discusiones tanto del medio profesional como académico. Sin embargo, los elementos cinéticos han estado presentes en la arquitectura desde sus inicios: puertas y ventanas pivotantes o deslizantes representan esta idea ya en las primeras construcciones, con el accionamiento manual, que en la actualidad tiende a reemplazarse por sistemas electrónicos e inteligentes. La arquitectura cinética consiste en la exploración de las variadas transformaciones físicas en los elementos que conforman un diseño. Esta presenta muy buenos resultados en el desempeño ambiental de las edificaciones, mejorando las condiciones de confort al controlar la interacción con el ambiente externo. Los sistemas cinéticos y responsivos considerados más avanzados surgen a finales del siglo XX e inicios del XXI, junto al desarrollo de la inteligencia artificial (Ramzy y Fayed, 2011).

Así como la piel en los organismos vivos, la envoltura de los edificios puede trabajar de manera adaptativa a los condicionantes climáticos locales. Con la evolución de las herramientas computacionales, de fabricación, automatización y robótica, es posible proyectar envolturas que ejerzan funciones más allá de la separación entre interior y exterior. Se pueden programar transformaciones físicas que adapten la edificación, por ejemplo, a las condiciones ambientales contemporáneas a la ocupación, a su patrón de uso a lo largo del tiempo, al comportamiento del usuario, etc. (Beesley y Khan, 2009; Linn et al., 2014). 
Según Ramzy y Fayed (2011), los sistemas cinéticos en la arquitectura pueden ser clasificados de acuerdo con su configuración, forma de control y utilidad final, y divididos en cuatro subcategorías: unidades de sistema de piel; elementos retractables; edificios pivotantes y sistemas biomecánicos. El primero trata de la división de elementos de la envoltura en pequeñas unidades móviles, con posibilidad de girar o moverse sin cambios significativos en la configuración del edificio. El segundo se refiere a elementos de la envoltura que son retráctiles, con posibilidad de doblarse o expandirse de acuerdo con la necesidad, provocando cambios significativos en la edificación. El tercero abarca edificaciones capaces de girar alrededor del propio eje, en general impulsadas a través de energía eólica o solar. El cuarto, y último, comprende sistemas que responden a aspectos ambientales, externos o internos a través de sensores y otros gatillos, constituyéndose estos en el objetivo final para sistemas cinéticos (Figura 1).
Los sistemas de elementos automatizados implican el uso de tres tipos de elementos: sensores para obtener información; un controlador para determinar los tipos de acciones; $y$, por último, diversos tipos de elementos mecánicos. Se trata de un enfoque que necesariamente es multidisciplinario al incluir conocimientos en el área de mecánica, eléctrica y de ingeniería de software (AlMasrani et al., 2018).

\section{MÉTODO}

El estudio combina dos procedimientos metodológicos, con etapas coherentes con sus respectivos objetivos, uno experimental exploratorio y otro cualitativo evaluativo. Primeramente, el procedimiento experimental

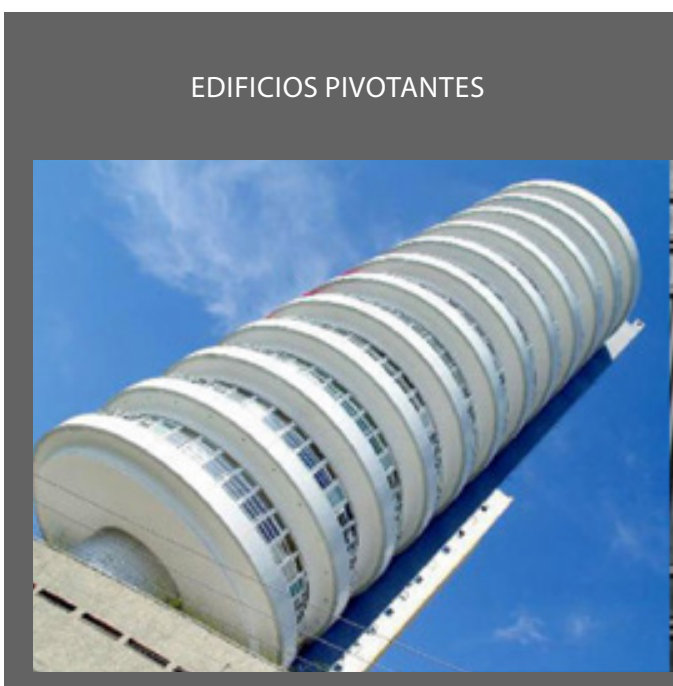

UNIDADES DE SISTEMA DE PIEL

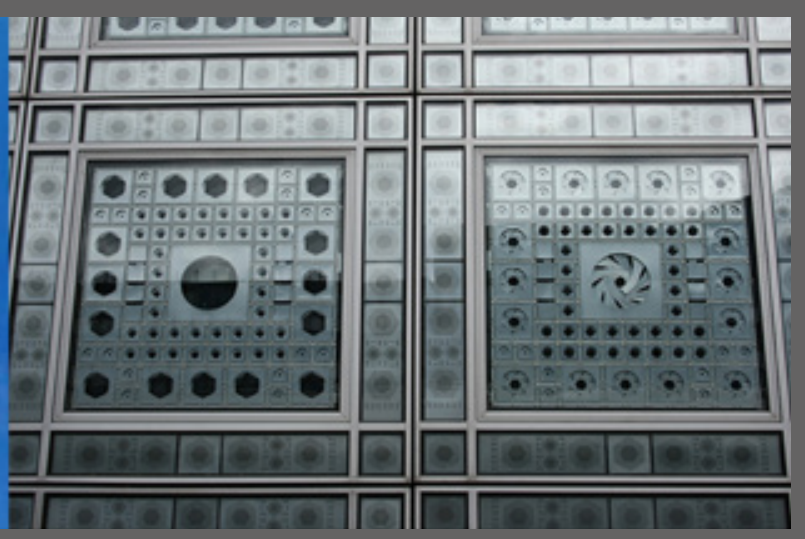

ELEMENTOS RETRÁCTILES

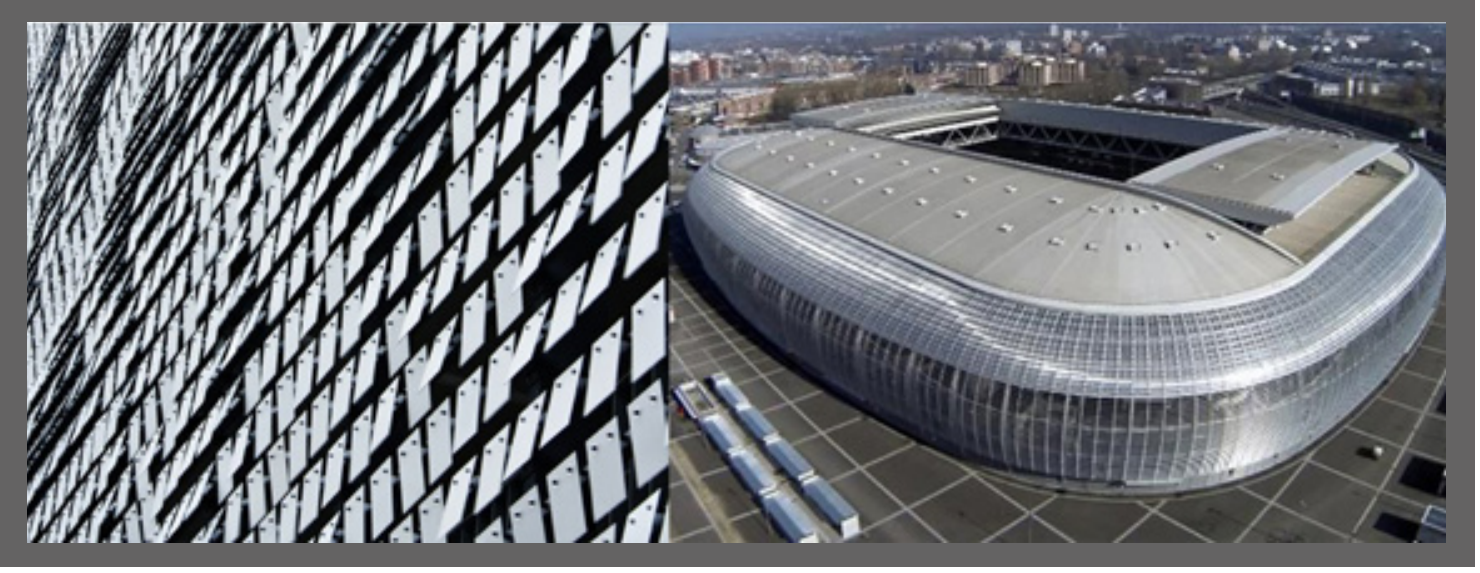

Figura 1 Sistemas cinéticos en la arquitectura. Fuente: Adaptado de Ramzy y Fayed (2011). 
explora y explicita el desarrollo del objeto de estudio. Este consiste en la elaboración de un modelo digital funcional y un prototipo físico de una envoltura formada por elementos huecos modulares responsivos al movimiento humano y a la trayectoria solar. En seguida, se propone el procedimiento cualitativo evaluativo, en la forma de un estudio piloto de aplicación del experimento con el uso combinado de un grupo focal. De esta manera, se busca averiguar el impacto en la enseñanza de la arquitectura, tanto de la aplicación del experimento desarrollado en esta investigación como, y consecuentemente, de las discusiones postuladas en el contexto teórico del artículo. Las etapas de elaboración apuntan a la utilización de abordaje, técnicas y materiales que posibiliten su replicación. Para este artículo se adoptaron las etapas metodológicas y procedimientos siguientes (Figura 2).

\section{Experimento:}

Estudio del módulo y la característica del movimiento. Para ello, se utilizaron revisiones bibliográficas, estudios prácticos y técnicas de prototipado físico.

Construcción de modelo digital funcional. Se emplearon técnicas de modelado algorítmico y paramétrico a través del plugin Grasshopper para Rhino 3D; lenguaje de script; y plugins que permiten la extracción de datos ambientales como el Ladybug, además de un plugin para la integración del modelo con un sensor de movimiento, el Firefly.

Identificación de medio para capturar el movimiento humano. Se consideraron sensores de movimiento $y$ escaneado accesibles, como el Kinect.

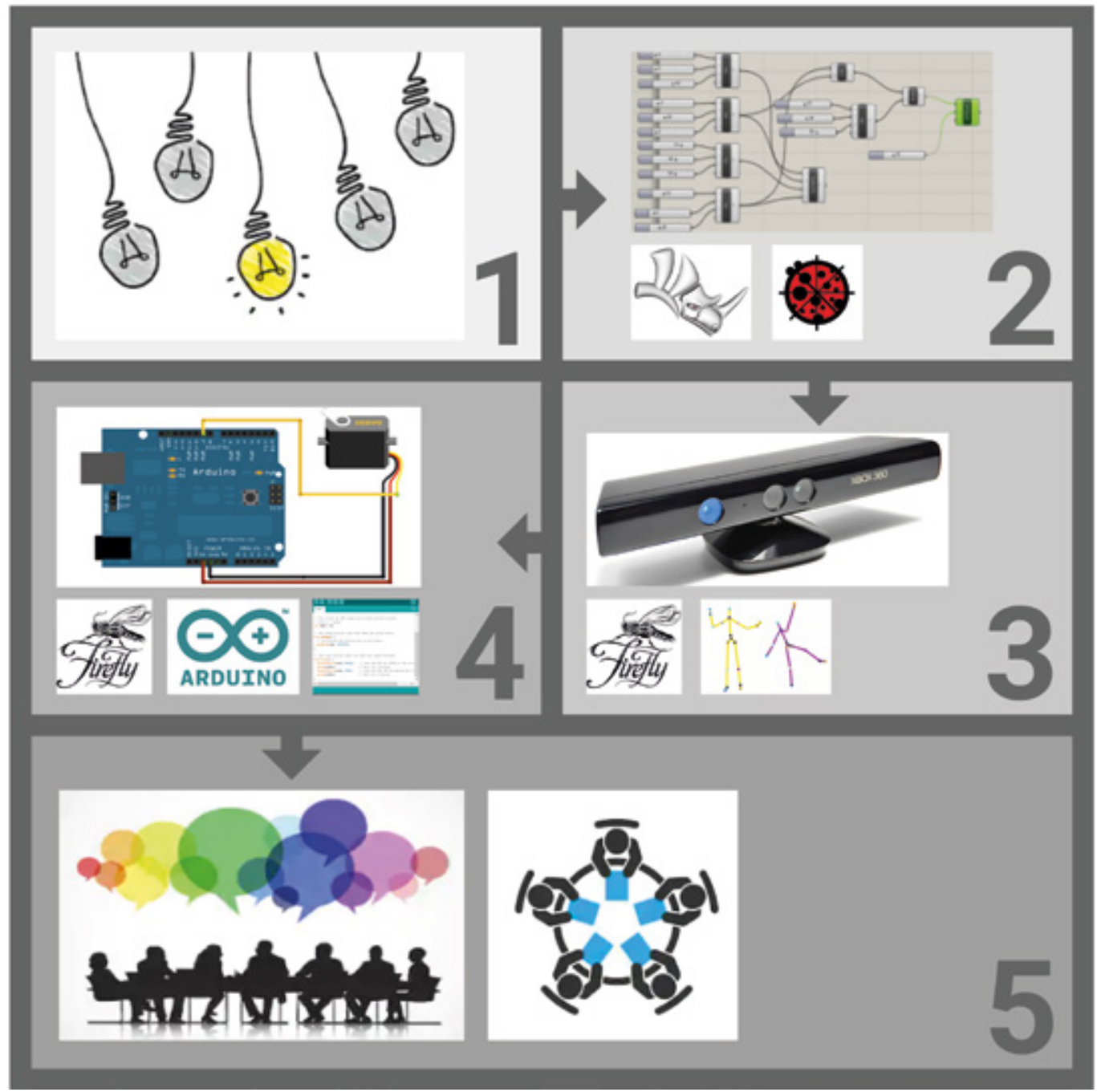

Figura 2 Etapas metodológicas. Fuente: Elaboración de los autores. 


\begin{tabular}{ccc}
\hline Sexo & Graduación & Post graduación \\
\hline Femenino & 2 & 2 \\
\hline Masculino & 2 & 1 \\
\hline
\end{tabular}

Tabla 1 Perfil de los participantes del taller.

Asociación del movimiento capturado con el modelo digital paramétrico y físico. Se contempló el uso de lenguaje de programación, construcción de modelo físico y uso de plataforma de prototipado electrónico, como el Arduino UNO. Como resultado, se obtuvo un prototipo funcional de bajo costo y la caracterización de una estructura conceptual para un ejercicio replicable en escuelas de arquitectura latinoamericanas.

\section{Estudio Piloto:}

Planeamiento y formación de un taller práctico con previsión de 8 horas de clase, con siete alumnos voluntarios de graduación y de postgrado en arquitectura y urbanismo (Tabla 1). Ejecución de la primera parte de un grupo focal con los voluntarios sobre el tema de investigación, donde se formularon preguntas para comprender la opinión y el conocimiento de los voluntarios sobre los temas de automatización y arquitectura cinética. Realización, en el taller, de un ejercicio de replicación individual por parte de los voluntarios del experimento desarrollado. Por último, cumplimiento de la segunda parte del grupo focal con los voluntarios, con el fin de entender oportunidades percibidas sobre los mismos temas del primer grupo focal y de visualizar perspectivas a futuro.

\section{RESULTADOS}

Conforme la descripción de los procedimientos metodológicos, los resultados son presentados, primeramente, en términos del experimento y sus respectivas cuatro etapas, $y$, a continuación, en términos del estudio piloto.

La primera etapa del experimento consistió en la ideación del prototipo, el establecimiento del módulo y la identificación del movimiento de la pared cinética. Para facilitar la aplicación del enfoque se creó un módulo prismático de $20 \times 20 \times 2 \mathrm{~cm}$ que gira alrededor del propio eje, funcionando como un elemento de sombreado horizontal. Dos accionamientos para el giro de los módulos fueron considerados: primero, el acercamiento y el movimiento humano; $y$, segundo, la posición del sol en relación a la pared (Figura 3).

La primera consideración busca la interactividad, la variación de la permeabilidad y la visión hacia el exterior de la pared, principalmente a la altura de los ojos. En este sentido, los módulos se accionan y se abren con la aproximación humana. Se procuró, desde el principio, que el accionamiento de los módulos siguiera el diseño del movimiento humano para intensificar la interacción. La segunda consideración, el accionamiento basado en la posición solar, está subordinada a la primera. Se utilizó el ángulo entre la "posición del sol" en la bóveda celeste y la base de la apertura de cada módulo para establecer su ángulo de apertura. Este accionamiento buscó el desempeño térmico a través del sombreado total del lado interior de la pared, además del control de fenómenos incómodos, como el de deslumbramiento.

En la segunda etapa, la construcción del modelo digital funcional, se emplearon técnicas de modelado algorítmico y paramétrico a través del plugin de programación visual Grasshopper, desarrollado para el software de modelado Rhinoceros 3D, y uso de lenguaje script. Para capturar los datos ambientales relacionados con el sol, se usó el plugin Ladybug para Grasshopper y, para capturar la silueta para el accionamiento humano, el plugin Firefly. Fueron adoptados dos pasos de desarrollo en esta etapa. El primer de ellos fue establecer el modelo de la pared de manera paramétrica. Los parámetros de entrada del script son la cantidad de módulos en el eje $x$ e y de la pared, tamaño de las estructuras de soporte, dimensiones de los módulos y espesor. También es posible modificar la variación del ángulo de giro de la apertura de los módulos (Figura 4).

El segundo paso consistió en establecer el área de influencia de accionamiento de los módulos a través del movimiento humano. Se utilizó, para ello, un principio de modelado paramétrico llamado "puntos atractivos". La silueta humana fue convertida en puntos que accionaban los módulos. Como constituían más de un punto, se realizó un script para asociar un grupo de módulos al punto atractivo más cercano de forma proporcional a la distancia con el punto respectivo. De esta forma, fue posible diseñar la pared, reproducir el movimiento humano y establecer un efecto "fade" en los bordes. Como parámetro de entrada, se estableció una distancia absoluta y otra relativa en relación a los puntos atractivos. Así, por ejemplo, es posible afirmar que el $20 \%$ de módulos serán accionados (parámetro relativo) y que los módulos con distancia mayor a 4 metros no serán accionados (parámetro absoluto) (Figura 5 y 6). En la definición de Grasshopper, este paso se estableció a través de funciones condicionales que se escribieron utilizando el lenguaje de secuencias de comandos en $\mathrm{C} \#$. 


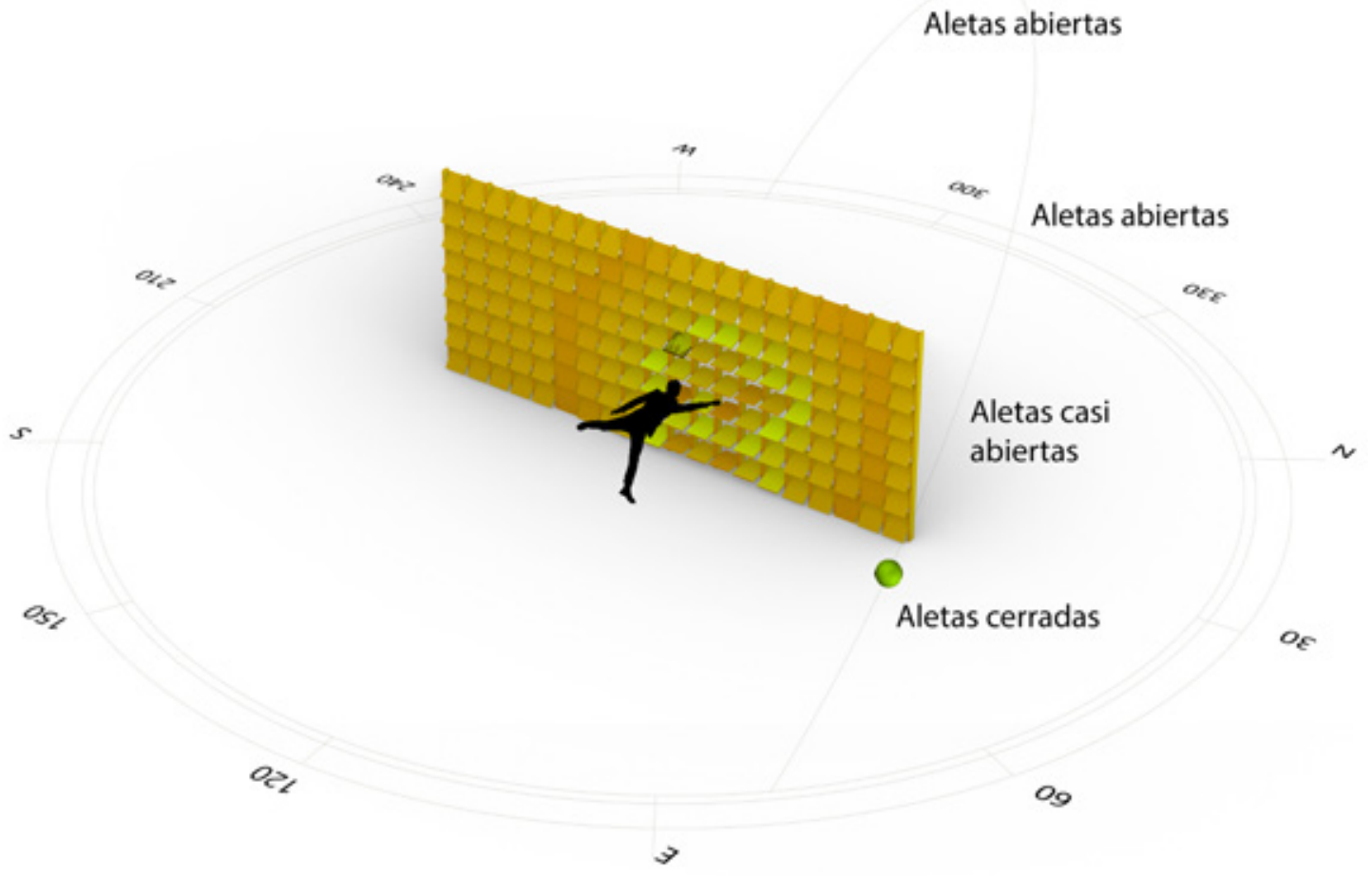

Figura 3 Creación de la pared cinética. Fuente: Elaboración de los autores.

Módulos accionados Indica el módulo Módulos accionados por el sol visualizado por el movimiento en el prototipo humano

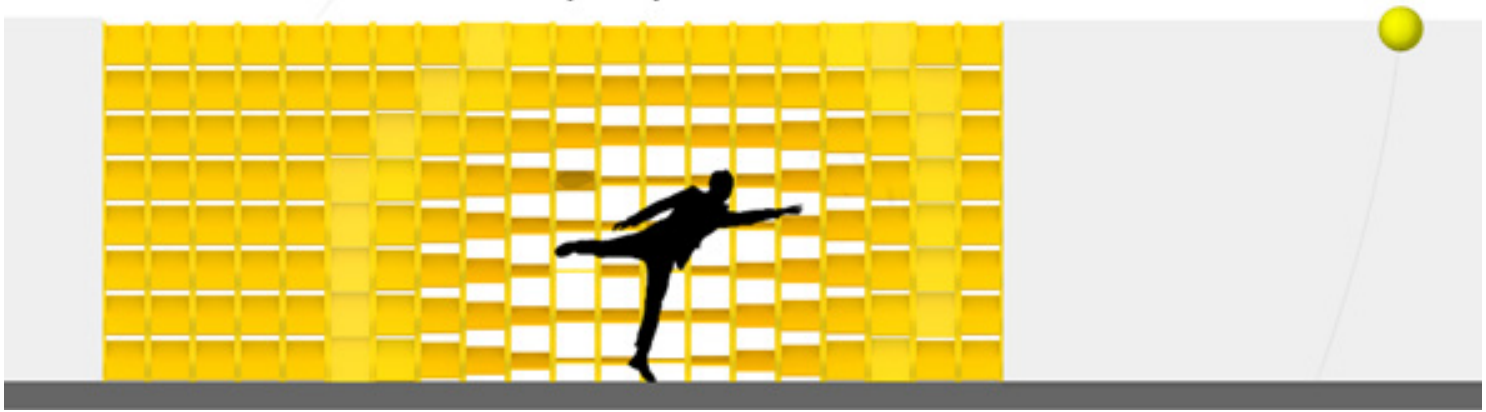

Figura 4 Geometría de la pared con módulos pivotantes. Fuente: Elaboración de los autores. 


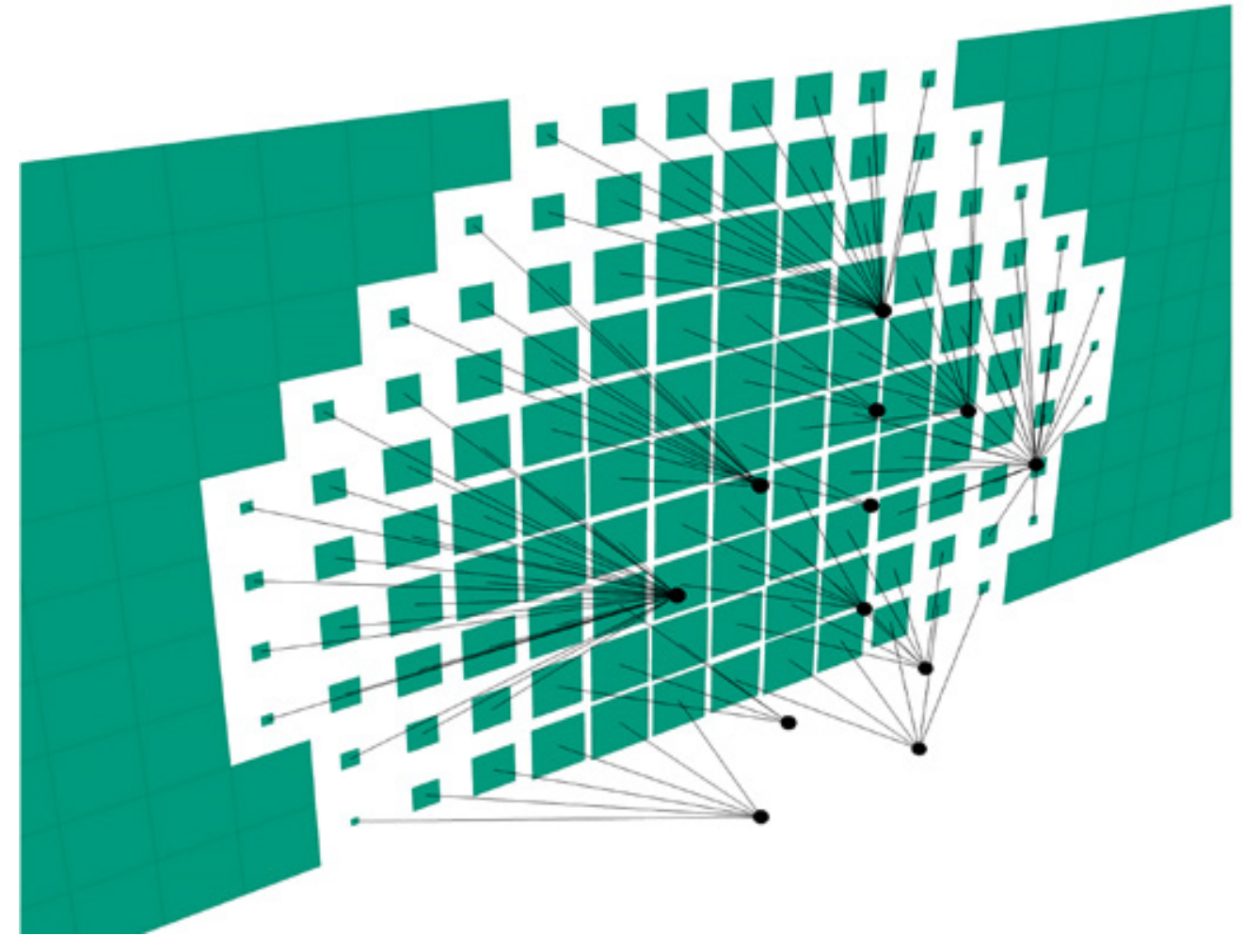

Figura 5 Asociación de módulos a los puntos escaneados. Área de influencia basada en la proximidad, y proporcional a la distancia. Fuente: Elaboración de los autores.

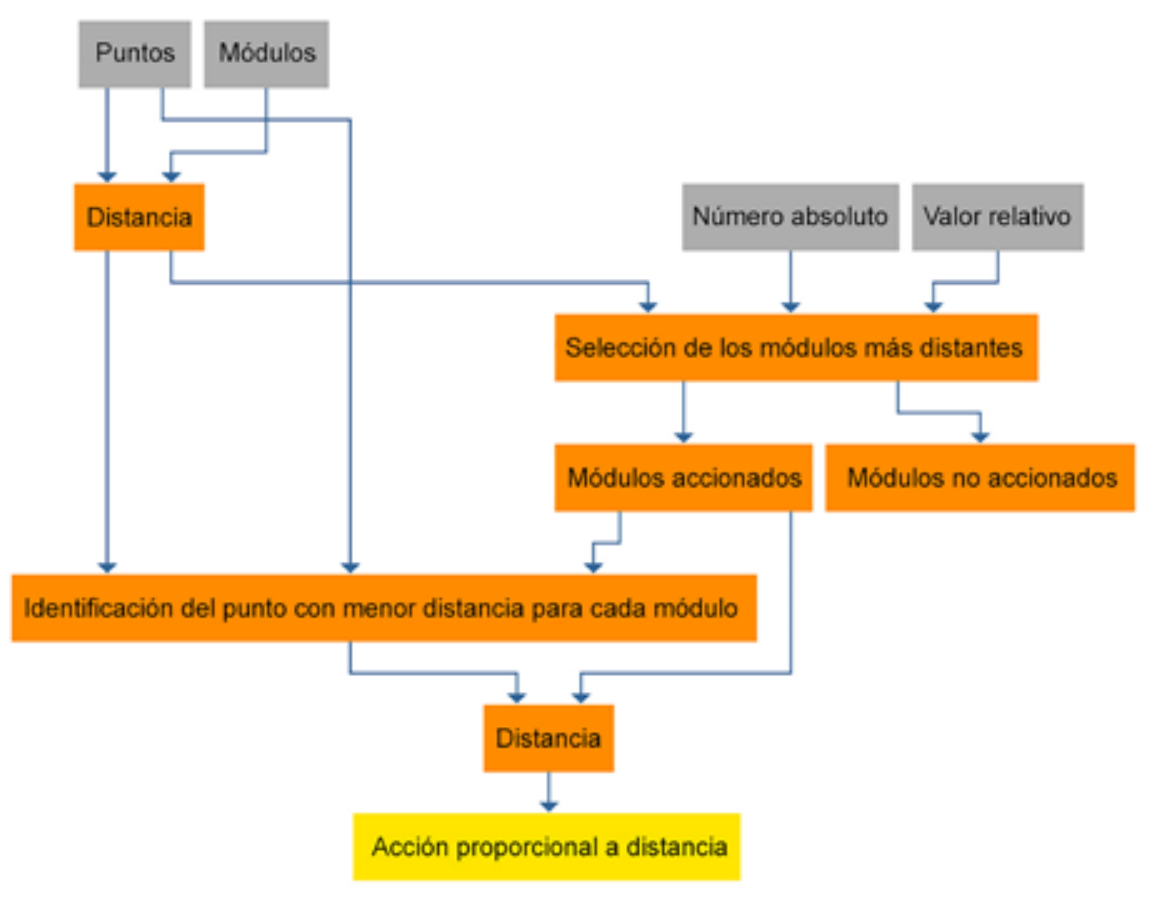

Figura 6 Taxonomía para la selección de los módulos activados. Fuente: Elaboración de los autores. 
La tercera etapa fue la de establecer los datos de la posición solar y la asociación con el ángulo de apertura de los módulos. Este paso utilizó un comando en C \# para definir la hora real del experimento. Como parámetro de entrada, además de la hora, el script usó la posición de la pared en relación al norte y la latitud y longitud de donde se encuentra la pared. A través del plugin Ladybug fue posible precisar el ángulo de la posición del sol en relación al módulo y también si éste se encontraba frente o atrás de la pared. Esta información fue relevante para que la pared fuera accionada por el sol sólo cuando estuviera frente a la pared, es decir, sólo cuando los módulos necesitasen ejercer la función de sombreado (Figura 7). Al final, el script desarrolló el funcionamiento que se presenta en la Figura 8.

En esta etapa también se estudiaron las posibilidades de utilización de sensores de movimiento y escaneado simplificado para realizar la captura del movimiento humano. Aquí fue evaluada la estrategia para la asociación de datos capturados con el modelo digital en tiempo real. Se adoptó, para el proyecto, el sensor de movimiento Kinect, en virtud de su alta disponibilidad en el mercado y la disponibilidad de integración con computadoras.

La cuarta etapa se desarrolló en dos pasos distintos. Primero, la construcción del prototipo físico funcional de un módulo en escala de 1:1. Se buscó, en este punto, adoptar estrategias de montaje convencional y empleo de materiales de bajo costo y fácil acceso. Con el propósito de probar la accesibilidad dos módulos, uno de ellos fue construido utilizando una hoja de papel pluma y, el otro, madera y espuma de poliestireno (Figura 9). El segundo paso involucró la asociación de los movimientos de uno de los módulos de la envoltura con el modelo físico. Dentro del script gráfico de Grasshopper se añadió la posibilidad de seleccionar un módulo específico para ver su funcionamiento en el prototipo físico. La obtención de la información del ángulo y el movimiento propiamente dicho se realizaron mediante una plataforma de prototipado electrónico, el Arduino UNO, y un servo motor. La comunicación entre la placa y el algoritmo se llevó a cabo a través del plugin Firefly.

Finalizado el desarrollo, exitoso por cierto, del experimento, se inició el estudio piloto con el taller de arquitectura cinética y un grupo focal.

Con el fin de profundizar el entendimiento de las oportunidades que la discusión de arquitectura cinética puede ofrecer a la enseñanza de arquitectura en América Latina, se condujo un taller, combinado con grupos focales, que abordó las temáticas vinculadas con este estudio, en una escuela de arquitectura brasileña. Dicho taller, con duración de ocho horas a partir del experimento desarrollado, fue estructurado

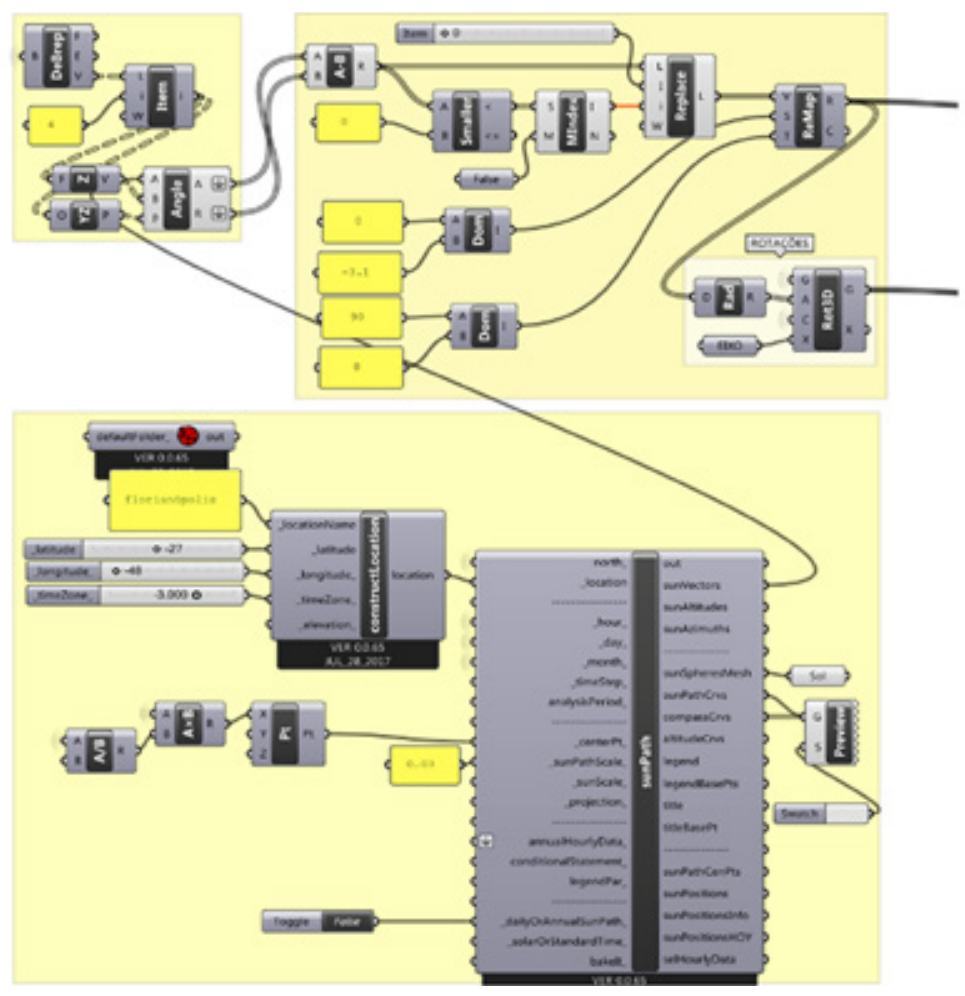

Figura 7 Script en Grasshopper que muestra la extracción de los datos ambientales a partir del archivo climático y de la hora local. Fuente: Elaboración de los autores. 


\section{PREPRINT}

AS54

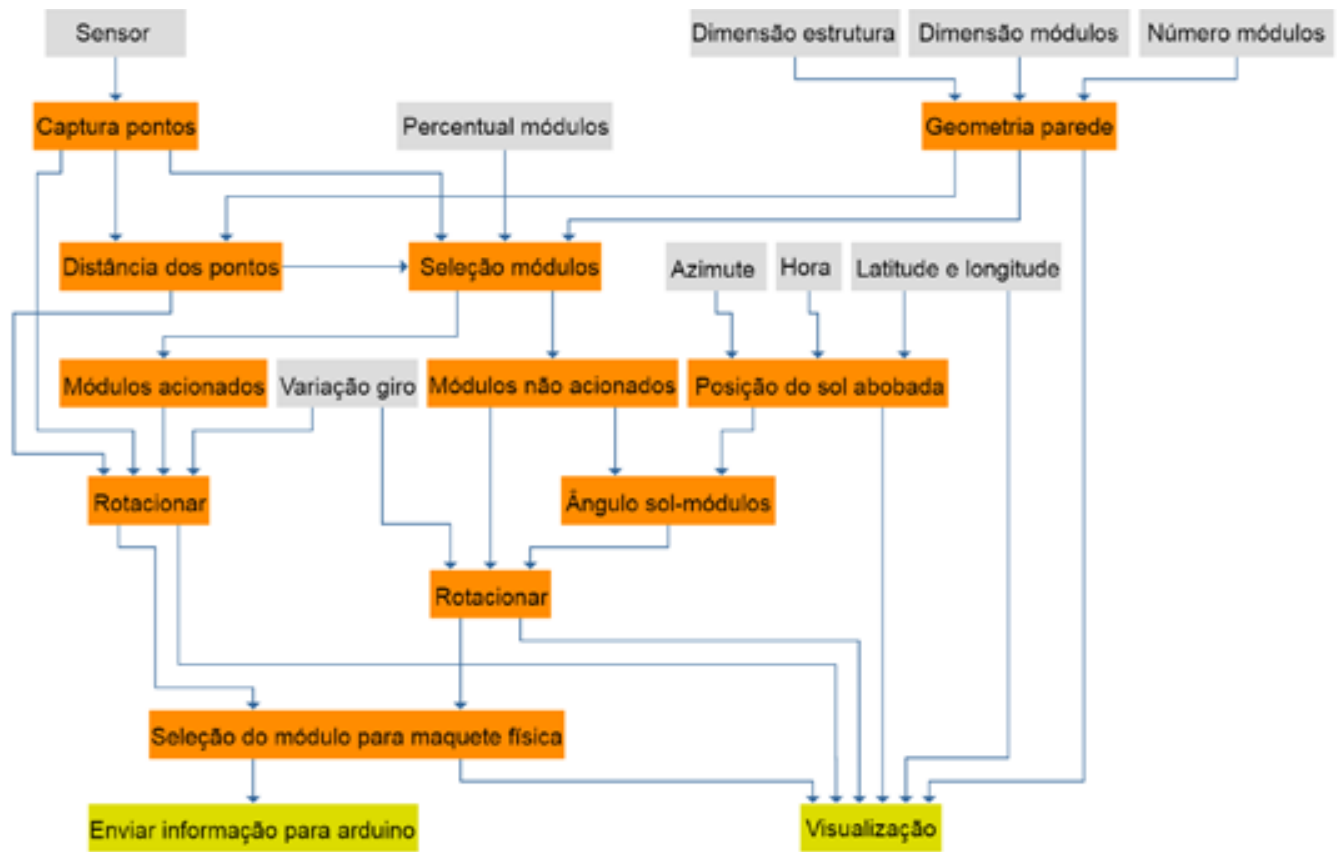

Figura 8 Taxonomía final del script. En gris, los parámetros de entrada; en naranja, las acciones de la secuencia de comandos y, en amarillo, los datos de salida. Fuente: Elaboración de los autores.

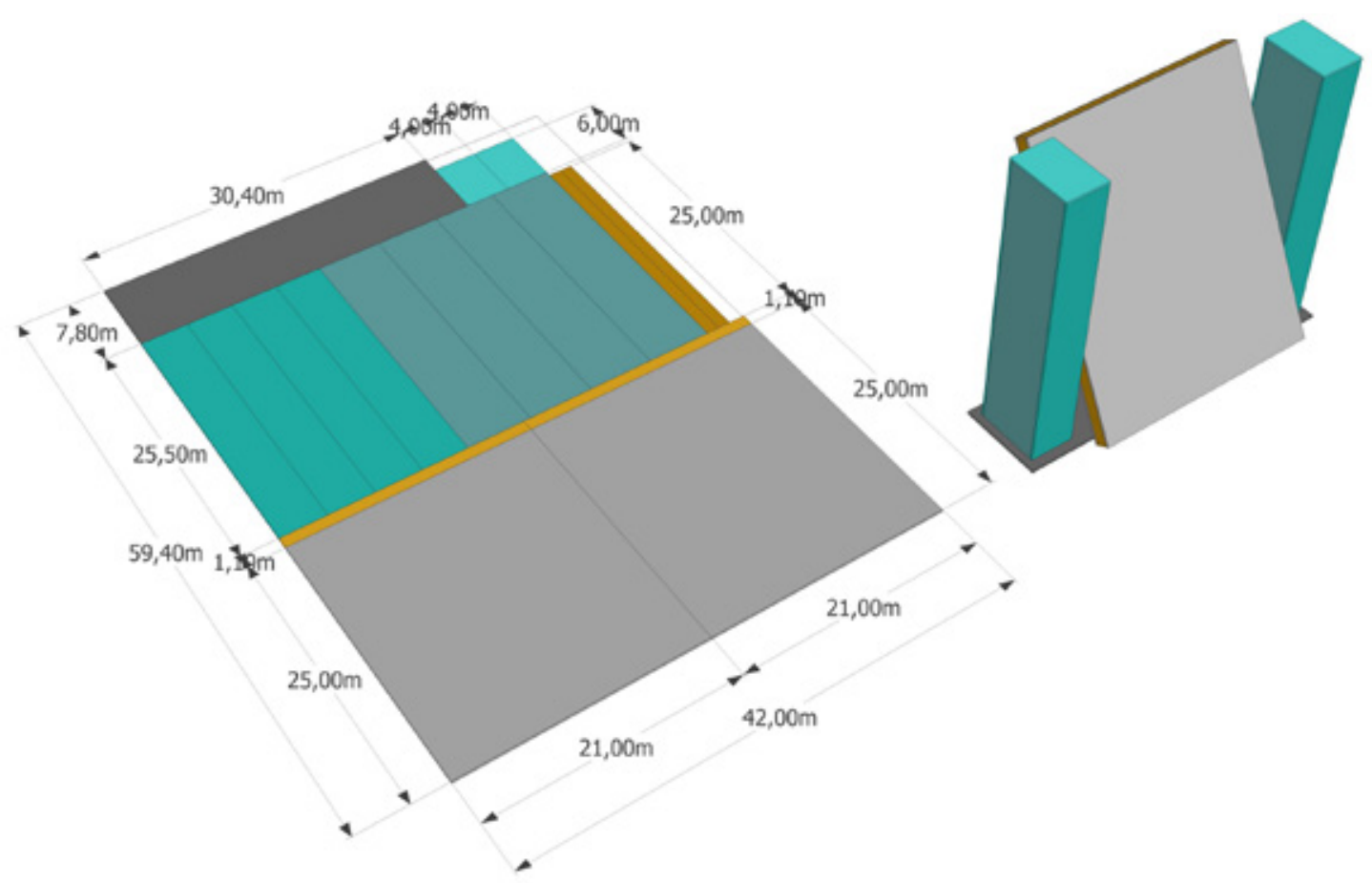

Figura 9 Planificación del prototipo de un módulo en escala de 1:1. Fuente: Elaboración de los autores. 


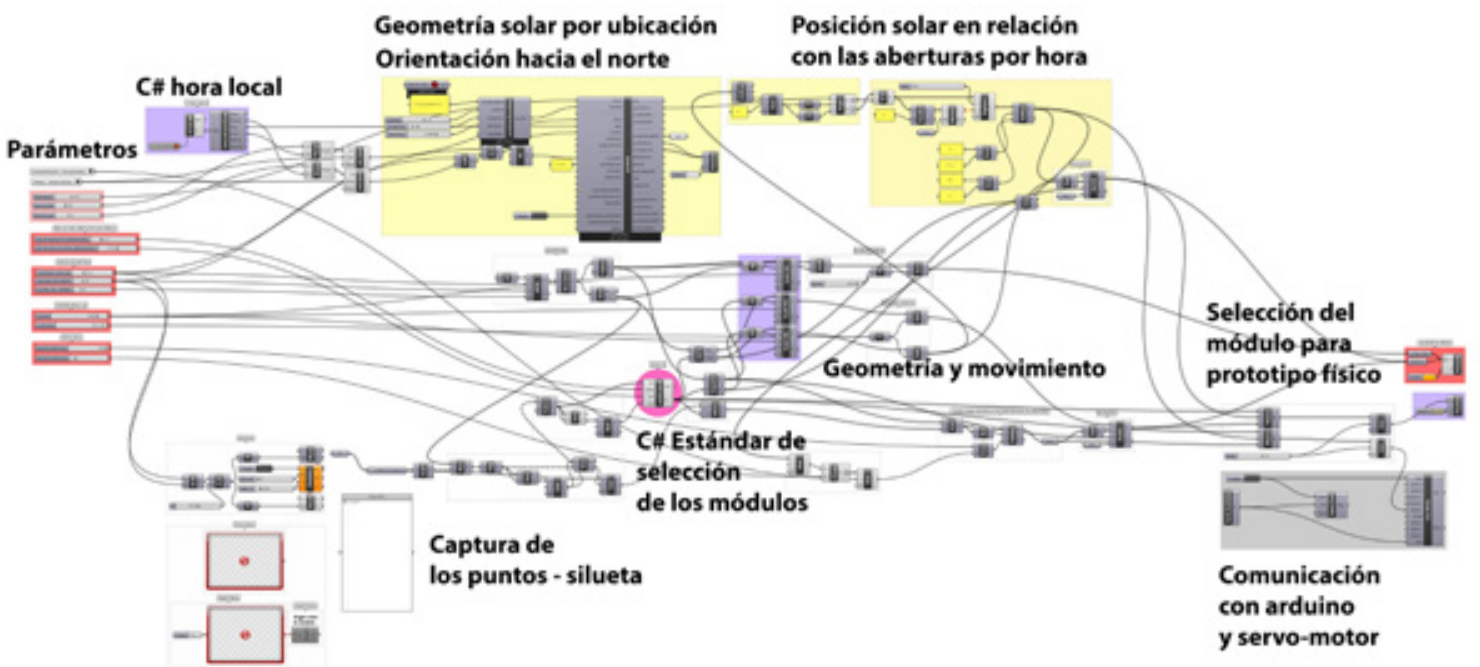

Figura 10 Apariencia del script final. Fuente: Elaboración de los autores.

como mecanismo de introducción de los participantes a las discusiones aquí expuestas. Así, el contenido del experimento se simplificó y dividió en tres momentos: la construcción de la definición de Grasshopper para el control de la pared cinética; la inserción en la definición del componente de detección a través del Kinect; $y$, la preparación del Arduino y la activación del mecanismo del módulo por servo motor. Para optimizar el tiempo del taller la pared cinética fue premontada por los investigadores.

Como resultado, todos los participantes del taller alcanzaron el producto final propuesto, independientemente del nivel de experiencia de ellos con las herramientas adoptadas. El grupo focal fue conducido para profundizar el entendimiento en el debate sobre el impacto de la inserción de la Arquitectura Cinética en la formación de arquitectos. Las actividades de este grupo fueron distribuidas en dos partes: una antes del taller, que se abocó a conceptos y experiencias previas con la tecnología; y otra, después del taller, que se centró en el acceso a la tecnología y en las oportunidades de enseñanza y aprendizaje respectivas. Ambos momentos fueron grabados digitalmente y transcritos para su posterior análisis formal de contenido. Participaron del taller siete alumnos de graduación y postgrado de la Universidade Federal da Paraíba, además de los investigadores autores del presente documento.
Como se discutió en los procedimientos metodológicos, la primera parte del grupo focal buscó de los participantes información sobre la comprensión previa de la noción de "arquitectura cinética" y sobre su experiencia con las herramientas propuestas para el taller, específicamente con Grasshopper, Arduino, Kinect y con dispositivos electrónicos por sensorización. En cuanto a las experiencias con las herramientas adoptadas, un participante señaló el uso de Grasshopper en combinación con Arduino y otros dos, su conocimiento de Grasshopper. El primer caso se trataba de una experiencia adquirida durante un intercambio en una universidad norteamericana. Según el relato, ésta fue positiva, sin embargo, limitada, pues consistió en replicación de definiciones y empleo de Arduino, previamente desarrollados sin autonomía para ajustes. Dicha práctica despertó el interés del participante que continuó explorando herramientas de programación gráfica en la Universidad Federal da Paraíba con apoyo de los investigadores. Los dos otros asistentes indicaron el uso de Grasshopper en el contexto del desarrollo de un análisis de la forma urbana, como parte de sus estudios de postgrado y cursos externos. Respecto a la comprensión del concepto de "arquitectura cinética", sólo un participante declaró comprensión parcial de tal concepto, advirtiendo únicamente la relación con el movimiento de mecanismos de edificios asociados al desempeño de funciones de protección solar. 


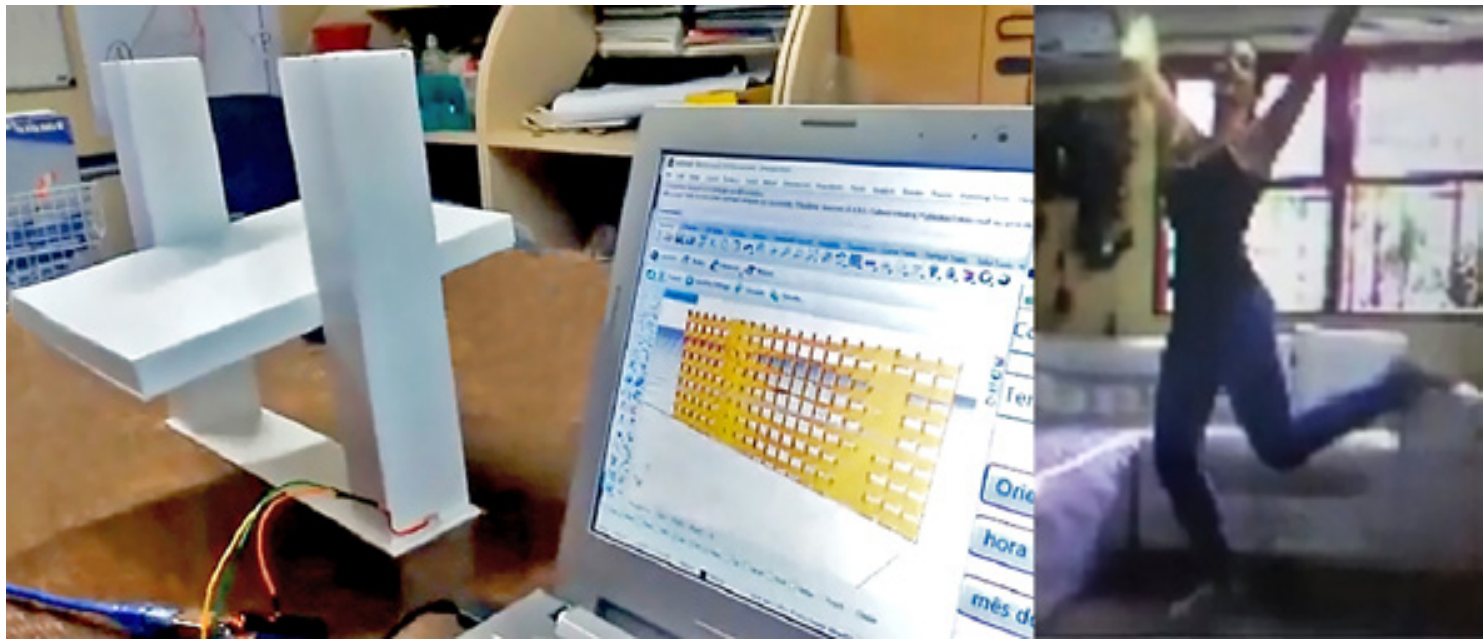

Figura 11 Interacción y prototipo físico y digital en funcionamento. Fuente: Elaboración de los autores.

Después del término del taller, la segunda parte del grupo focal fue llevada a cabo. Aquí se abordó la percepción de los participantes en relación al acceso a las tecnologías presentadas en el taller. Los estudiantes manifestaron que las tecnologías eran accesibles para aplicaciones de naturaleza académica a escala reducida, con materiales de bajo costo. Sin embargo, demostraron escepticismo en cuanto a la viabilidad de las aplicaciones en la escala del edificio. Este punto de vista se basó en tres argumentos: limitaciones de acceso a la tecnología adecuada para aplicaciones en escala 1:1 y en cantidades mayores; especulaciones sobre el coste añadido a la construcción, lo que, en consecuencia, destinaría estas tecnologías únicamente a una élite de consumidores; $y$, por último, preocupaciones por la falta de una cultura de mantenimiento preventivo y correctivo de sistemas como el propuesto. El contrapunto a esta concepción fue la discusión sobre eventuales reducciones en costos operativos derivadas de la mejora del desempeño térmico en función de sistemas cinéticos. Se hizo evidente la distinción de la relación costo-beneficio para el contexto académico y el mercado actual de la construcción. Se destacó, asimismo, la importancia de explotar estas tecnologías en el desarrollo de sistemas con costos más adecuados a la realidad brasileña. Por consiguiente, se reforzó la importancia de la inserción del tema en el ámbito de la investigación y la enseñanza en arquitectura. Este enfoque asociado a procesos de simulación, modelado y prototipado rápido permitiría reducir los costos y la resistencia a la implementación de dichas tecnologías.

Cuando se indagó sobre las oportunidades de enseñanza y aprendizaje con tecnologías, como las abordadas en el taller, hubo consenso en que servirían como preparación para etapas más avanzadas, para aplicaciones cinéticas y/o aplicaciones diversas de sensores. De la misma forma, y puntualmente, se reconoció que la asociación de estos temas a disciplinas de inicio o mediados de la carrera de arquitectura permitiría un tiempo mayor de maduración del conocimiento en el transcurrir de la formación de un arquitecto. Desde esa perspectiva, los participantes entendieron que los alumnos de arquitectura estarían preparados para adoptar soluciones como éstas de manera integral en sus propuestas y no sólo como elementos a ser añadidos después del desarrollo del proyecto. La desmitificación de la tecnología fue también apuntada como oportunidad de acercamiento, lo que podría concretarse a través de cursos de ingeniería, automatización, diseño industrial y computación, pues posibilitaría la capacitación de todos los involucrados en el desarrollo de proyectos integrados.

La toma de conciencia de que estos enfoques en arquitectura constituyen una realidad presente en otros países fue asumido como un refuerzo a la demanda por el desarrollo de tales orientaciones en la enseñanza de la disciplina en Latinoamérica. Se destacaron, entonces, las proposiciones de desarrollos asociados a la interactividad del hombre con el objeto, a la relación del espacio con la calidad ambiental y, por fin, a la integración de proyectos. Las discusiones del grupo focal concluyeron con la identificación de proyectos que podrían ser explotados para el desarrollo de prototipos análogos al del taller. Los temas de automatización residencial fueron preteridos por temas de proyecto vinculados a escenografía, edificios de función cultural y edificios educacionales; todos como manera de difundir las oportunidades generadas por este abordaje a un público más amplio.

\section{CONCLUSIONES}

Como se expuso en este artículo, la presente investigación se propuso estructurar conceptualmente el proyecto y el prototipo funcional de una pared cinética responsiva a las necesidades de los seres humanos en términos de confort térmico y de su relación con la trayectoria solar. Por medio de los procedimientos presentados aquí, y a partir de un equipo interdisciplinario 
colaborativo de arquitectos investigadores, fue posible el desarrollo adecuado del experimento. De la misma manera, una vez comprendido su funcionamiento, fue posible convertir el experimento en un ejercicio académico a ser aplicado en un taller de arquitectura. En un primer nivel, se logró la viabilidad de aproximaciones entre temas como arquitectura responsiva a la enseñanza de arquitectura y urbanismo. En este artículo, tal aproximación fue posible por medio del uso de modelado paramétrico asociado al modelado algorítmico en el diseño de elementos de envolturas. Mediante el desarrollo de un experimento construido para este contexto se demuestra la posibilidad de integración teórica y práctica desde una óptica contemporánea de proyecto arquitectónico.

Limitándose al ámbito propuesto por la investigación, los resultados indicaron un potencial en el marco de la investigación. Claramente, se reconoció eventuales parcialidades de los voluntarios. Sin embargo, se notó un alineamiento importante entre los resultados del grupo focal y los discursos teóricos que circundan el tema. Futuras investigaciones deben considerar replicaciones en otras escuelas de arquitectura en Latinoamérica, así como a partir de experimentos distintos. Se vislumbra, en ese sentido, la aplicación de tales ejercicios, inicialmente en disciplinas de proyectos especiales, y la construcción de una inserción definitiva en currículos de enseñanza de arquitectura y urbanismo. Asimismo, avances paralelos pueden refinar el desarrollo de prototipos en escala 1:1 de este sistema, o de sistemas análogos, dentro del contexto de viabilidad, en sus respectivas localidades.

No obstante, se admite que las aproximaciones alcanzadas entre arquitectura responsiva y modelado paramétrico asociado al modelado algorítmico en el diseño de elementos de envolturas, no son oportunidades disponibles para todo arquitecto en formación hoy. Efectivamente, de un total de más de setecientas escuelas de arquitectura en Brasil, ien cuántas de ellas la temática foco de este artículo participa del currículo? No es un riesgo afirmar que en una gran mayoría estos temas no participan. Existe, por ende, una falta de sincronía entre las preocupaciones contemporáneas y la base curricular del arquitecto que actuará en los próximos años en la construcción de nuestras ciudades. Se apuesta, desde esta óptica, que, específicamente, la combinación de temáticas como diseño basado en el performance (Oxman, 2008; Beesley y Khan, 2009) y métodos de proyecto auxiliados por computador asociados a la programación (Kotnik, 2010), en el contexto de la creciente popularización de laboratorios de fabricación digital (Sperling, 2015), constituye una oportunidad disruptiva importante. Como tal, ésta necesita ser investigada en su totalidad y en sus diversas variantes.

En esa dirección, la investigación presentada contribuye pedagógicamente a la discusión de la complejidad del proyecto de envolturas y a la introducción de temas como automatización, modelado paramétrico y programación en ejercicios de diseño contemporáneo. Esta respuesta está alineada con las discusiones presentadas en la introducción de este artículo. De esta manera, también, este artículo pudo abordar las posibilidades de quiebre, de lo que Kotnik (2010) define como problemas de adaptabilidad e inercia del sistema educativo.

\section{REFERENCIAS BIBLIOGRÁFICAS}

BEESLEY, Philip; KHAN, Omar. Responsive Architecture/ Performing Instruments. Architectural League of New York, 2009.

LINN, Charles. Kinetic architecture: design for active envelopes. Melbourne: Images Publishing, 2014.

FOX, Michael (ed.). Interactive Architecture: Adaptive World. New York: Chronicle Books, 2016.

KOTNIK, Toni. Digital architectural design as exploration of computable functions, International journal of architectural computing, 2010, vol. 8, $n^{\circ} 1$, pp. $1-16$.

KRONER, Walter M. An intelligent and responsive architecture, Automation in construction, 1997, vol. 6, $\mathrm{n}^{\circ}$ 5-6, pp. 381-393.

AL-MASRANI, Salwa M.; AL-OBAIDI, Karam; ZALIN, Nor e ISMA, M. Aida. Design optimisation of solar shading systems for tropical office buildings: Challenges and future trends, Solar Energy, 2018, vol. 170, pp. 849-872.

JAGGER, Robert W. New options for the envelope. Face Time: the emergence of the facade as the integrative factor in holistc building design. FACADE TECTONICS: World Congress 2016. Los Angeles. Tectonic Press, 2016, pp. 1 - 14

OXMAN, Rivka. Digital architecture as a challenge for design pedagogy: theory, knowledge, models and medium, Design studies, 2008, vol. 29, n² 2, pp. 99-120.

RAMZY, Nelly y FAYED, Hatem. Kinetic systems in architecture: New approach for environmental control systems and context-sensitive buildings, Sustainable Cities and Society, 2011, vol. 1, n 3, pp. 170-177.

SPERLING, David M.; HeRRERA, Pablo C.; CELANI, Gabriela y Scheeren, Rodrigo. Fabricação digital na América do Sul: um mapeamento de linhas de ação a partir da arquitetura e urbanismo. São Paulo. Blucher Design Proceedings, 2015, pp. 119-125. 